Nonlinear Processes in Geophysics, 12, 741-753, 2005

SRef-ID: $1607-7946 / \mathrm{npg} / 2005-12-741$

European Geosciences Union

(C) 2005 Author(s). This work is licensed

under a Creative Commons License.

\title{
Role of the hydrological cycle in regulating the planetary climate system of a simple nonlinear dynamical model
}

\author{
K. M. Nordstrom ${ }^{1}$, V. K. Gupta ${ }^{2,3}$, and T. N. Chase $^{2,4}$ \\ ${ }^{1}$ Colorado Research Associates, 3380 Mitchell Lane, Boulder, Colorado 80301, USA \\ ${ }^{2}$ Cooperative Institute for Research in the Environmental Sciences, University of Colorado, Boulder, Colorado 80309, USA \\ ${ }^{3}$ Department of Civil and Environmental Engineering, University of Colorado, Boulder, Colorado 80309, USA \\ ${ }^{4}$ Department of Geography, University of Colorado, Boulder, Colorado 80309, USA
}

Received: 12 July 2004 - Revised: 15 March 2005 - Accepted: 16 March 2005 - Published: 29 July 2005

Part of Special Issue "Nonlinear deterministic dynamics in hydrologic systems: present activities and future challenges"

\begin{abstract}
We present the construction of a dynamic area fraction model (DAFM), representing a new class of models for an earth-like planet. The model presented here has no spatial dimensions, but contains coupled parameterizations for all the major components of the hydrological cycle involving liquid, solid and vapor phases. We investigate the nature of feedback processes with this model in regulating Earth's climate as a highly nonlinear coupled system. The model includes solar radiation, evapotranspiration from dynamically competing trees and grasses, an ocean, an ice cap, precipitation, dynamic clouds, and a static carbon greenhouse effect. This model therefore shares some of the characteristics of an Earth System Model of Intermediate complexity. We perform two experiments with this model to determine the potential effects of positive and negative feedbacks due to a dynamic hydrological cycle, and due to the relative distribution of trees and grasses, in regulating global mean temperature. In the first experiment, we vary the intensity of insolation on the model's surface both with and without an active (fully coupled) water cycle. In the second, we test the strength of feedbacks with biota in a fully coupled model by varying the optimal growing temperature for our two plant species (trees and grasses). We find that the negative feedbacks associated with the water cycle are far more powerful than those associated with the biota, but that the biota still play a significant role in shaping the model climate. third experiment, we vary the heat and moisture transport coefficient in an attempt to represent changing atmospheric circulations.
\end{abstract}

\section{Introduction}

Earth's climate has remained surprisingly stable since its formation four and a half billion years ago, a situation which has led to the hypothesis that the Earth Climate System (ECS)

Correspondence to: K. M. Nordstrom

(knordstrom@ comcast.net) is a self-regulating complex system. It has been proposed (Lovelock, 1972; Lovelock and Margulis, 1974) that the biosphere plays a key role in this climatic self-regulation. However, the presence of water on earth was a prerequisite for life to have originated, and for it to have evolved since then. Indeed, water is the fundamental substance supporting the existence of all life on earth and connecting it to the environment. The global hydrological cycle involving the movement of fresh water through the complete planetary system and the coexistence of water in three phases (liquid, solid and vapor) distinguishes Earth from apparently lifeless planets such as Mars and Jupiter. These broad observations suggest that the water cycle plays a key role in climatic self-regulation on earth with or without the existence of a biosphere, and serve as a focus for this article.

We will demonstrate, using a relatively simple nonlinear dynamical model, that the presence of an active water cycle on earth can play a key role in the regulation and evolution of its temperatures. Oceans act as low-albedo heat sinks and, in conjunction with the atmosphere, circulate to bring heat to the poles. Polar ice caps reflect a large percentage of incoming radiation. Clouds block both short-wave radiation entering the atmosphere and long-wave radiation leaving it, while precipitation and evaporation processes move heat and water between the ground, oceans and the atmosphere. Evapotranspiration processes couple all of this to biota, which in turn are an important contributor to the carbon cycle. These and other global bio-geochemical processes consist of positive and negative nonlinear feedbacks, and provide a basis for understanding the role of the hydrological cycle in the self-regulation of planetary temperatures.

Our simple nonlinear model, called a Dynamic Area Fraction Model (DAFM), has zero spatial dimensions (0-D) (Nordstrom et al., 2004; Nordstrom, 2002). It is a box model, in which the key biophysical processes involving the water cycle interact dynamically. The boxes of this model expand or contract dynamically as a result of these interactions. The 0 -D structure of the model greatly simplifies specification 
of spatial variabiltiy of biophysical processes and forcings. It allows us to test to what degree they contribute to the self-regulation of the planetary climate.

Although it is possible to generalize the DAFM framework to include a larger number of boxes than considered here, or to explicitly include spatial dimensions, we believe that it is necessary to first understand the role of the key dynamical processes involved in climatic self-regulation in their simplest form. Insights in this paper will provide a foundation on which to base further generalizations. Our motivation for this work is to understand all zero-order dynamical processes and their interactions through the water cycle while still maintaining maximum simplicity. The complexity of a climate model increases with added spatial dimensions, additional processes, or boxes, all of which increase the number of interacting parameters. Many of these parameters are poorly known. A complete error analysis or even a consistent interpretation of cause and effect becomes more difficult as model complexity increases.

On the other hand, models with low spatial dimensions and a small number of boxes have been used quite successfully in a variety of climate applications (Budyko, 1969; Paltridge, 1975; Watson and Lovelock, 1983; Ghil and Childress, 1987; Harvey and Schneider, 1987; O'Brian and Stephens, 1995; Houghton et al., 1995; Svirezhev and von Bloh, 1998). However, they generally lack many of the basic interactions which define our climate system. These can include an interactive hydrological cycle, cryosphere, biosphere, and biogeochemical cycles. We will present the construction of a DAFM representing a new class of 0-D model of an earth-like planet with coupled parameterizations for all the major components of the hydrological cycle involving liquid, solid and vapor phases. This model therefore shares some of the characteristics of an Earth System Model of Intermediate complexity (EMICS) (Claussen et al., 2002). We leave the addition of a dynamic carbon feedback for future work, though the carbon greenhouse effect is included in static form.

We begin by describing the construction of our model in Sect.2, and discuss feedbacks and parameter estimation in Sects. 3 and 4, respectively. In Sect. 5, we present two simple experiments with this model and the results for each. In Sect. 6, we conclude.

\section{Model description}

A model of even this "limited" complexity contains a relatively large number of variables, and for this discussion consistent naming conventions are important for clarity. Section 2.1 describes these conventions. Representing such a complex system with a DAFM requires establishing dynamics for energy balance and for other intrinsic variables, and it requires adding area fraction dynamics for oceans and ice caps. We shall describe the representation of these components as well as other aspects of the hydrological cycle in Sects. 2.2-2.6.

\subsection{Naming conventions}

This model consists of 6 regions and subregions, and local variables associated with each region are labelled with a subscript $i$ to denote regional dependence. This subscript can take the value $t$ for trees, $g$ for grasses, $d$ for bare ground ("dirt"), $o$ for ocean, $a c c$ for accumulation zone, or $a b l$ for ablation zone. If the subscript refers to the ice sheet as a whole, it is denoted by a capital letter $I$. Global mean quantities without regional dependence are denoted by an overbar, as in $\bar{T}$.

On each region, there are four prognostic variables to update. These are the region size $a_{i}$, the temperature $T_{i}$, the precipitable water $w_{i}$, and the soil moisture $s_{i}$. Each region also has several diagnostic variables associated with it, each of which affects the update for the prognostic variables. These diagnostic variables include precipitation $P_{i}$, evaporation $E_{i}$, cloud albedo $A_{c i}$, greenhouse greyness $v_{i}$, and cloudiness $a_{c i}$.

Finally, each region includes a transport term for each of the three transverse fluxes, heat, atmospheric moisture, and soil moisture. These are denoted by $F_{H i}$, where $H$ is the flux in question. The DAFM adjustment terms $K_{H i}$, which will be introduced below and are necessary to establish global conservation of quantities $H$, follow a similar convention.

\subsection{Energy balance}

The DAFM framework used here was introduced in Nordstrom et al. (2004) to remove the assumption of perfect local homeostasis through the albedo-dependent local heat transfer equation in the Daisyworld model of Watson and Lovelock (1983). It was pointed out by Weber (2001) that the regulation of local temperatures in Daisyworld, or homeostasis, was proscribed by the biota independently of solar forcing. Additionally, the strength of the model's homeostatic behavior, its main result, was forced to depend on an arbitrary parameter associated with the heat transport. The DAFM formulation enabled us to interpret the Daisyworld heat transport parameter physically, thus removing the artificiality in its parameter set. This representation also resulted in global temperature self-regulation similar to that in Daisyworld, despite the removal of the assumption of perfect local homeostasis. Under the DAFM framework used in Nordstrom et al. (2004), we stipulated local temperature adjustments due to solar radiative forcing in each box, and constrained the total energy of the planet to satisfy energy conservation. We will follow the same approach here for each box of our DAFM climate model.

We use a constant heat capacity across the system, $c_{p}$ in the energy balance equation, rather than differentiating it between land and ocean boxes. Estimates of $c_{p}$ vary widely (Harvey and Schneider, 1987) for both ocean and land, but we take $c_{p}=3 \times 10^{10} \mathrm{~J} / \mathrm{m}^{2} \mathrm{~K}$ as a representative value. Accounting for variations in $c_{p}$ by region will be addressed in future versions of the model. The results of the model in its steady state are relatively insensitive to the value of $c_{p}$, 
though integration paths in time vary, since $c_{p} d t$ provides an effective timescale for temperature update.

Regional temperatures in the model update through radiative balance

$c_{p} \dot{T}_{i}=R_{i n, i}-R_{\text {out }, i}+F_{T i}+K_{T i}$

in which $R_{i n}$ is the net incoming shortwave radiation at the surface, $R_{\text {out }}$ is the net outgoing longwave radiation from the surface, $F_{T i}$ represents the interregional heat transfer, $K_{T i}$ is the DAFM term explained below in Eqs. (9) and (10). $\dot{T}_{i}$ represents a total derivative of temperature in time. These quantities are defined to be

$$
\begin{aligned}
R_{\text {in }, i} & =S L A_{i}^{\prime}\left(1-a_{c i} A_{c i}\right) \\
R_{\text {out }, i} & =\sigma\left[\left(1-a_{c i}\right)\left(1-v_{i}\right) T_{i}^{4}+a_{c i} T_{c}^{4}\right] \\
F_{T i} & =D_{T}\left(\bar{T}-T_{i}\right)
\end{aligned}
$$

with $S$ the insolation of the present-day earth averaged over a sphere, $L$ the ratio of the model insolation to $S, A_{i}^{\prime}$ the local mean co-albedo for a region, $a_{c i}$ the region mean cloudiness, $A_{c i}$ the region mean cloud albedo, $\sigma$ the Stefan-Boltzmann factor for blackbody radiation, $1-v_{i}$ the greenhouse factor in the longwave greybody term, $T_{c}$ the cloud-top temperature, and $c_{p}$ the column-integrated heat capacity per $m^{2}$ of the earth. $D_{T}$ is a coefficient of heat transfer, and the form of $F_{T i}$ is used for simplicity (Budyko, 1969). While it is clear that using a single coefficient $D_{T}$ for every region of the model is technically not accurate, it is also clear that we must do so in order that the $F_{T i}$ terms integrate to zero over the globe. The error thus introduced should be investigated in a future model by using a more realistic representation of heat transport. It is important to note that in a DAFM, $R_{i n, i}$, $R_{\text {out }, i}$, and $F_{T i}$ are terms with specific physical significance, whereas $K_{T i}$ is present for accounting purposes.

\subsection{Dynamic areas}

The update of local variables in a DAFM is relatively straightforward, since it is a box model, except for the addition of an extra term to account for the movement of local boundaries. This term incorporates the adjustment of locallyconserved quantities like water mass and energy, and it performs an instantaneous averaging over the region.

To understand the term, consider a system with two regions of areas $a_{1}$ and $a_{2}=1-a_{1}$; and temperatures $T_{1}$ and $T_{2}$. The heat capacity for the system is $c_{p}$, so the total heat of the system is $Q=Q_{1}+Q_{2}=c_{p} a_{1} T_{1}+c_{p} a_{2} T_{2}$. Now let region 1 expand by $\delta a_{1}$ into region 2 , without the addition of any more heat from outside the system, and without accounting for any entropy effects from birth-death processes.

If we were to calculate energy balance with no correction to account for regional expansion, the new heat of region 1 would be

$Q_{1}^{\prime}=c_{p}\left(a_{1}+\delta a_{1}\right) T_{1}$

and the new heat of region 2 would be be

$Q_{2}^{\prime}=c_{p}\left(a_{2}-\delta a_{1}\right) T_{2}$ such that

$$
\begin{aligned}
Q^{\prime} & =Q_{1}^{\prime}+Q_{2}^{\prime}=c_{p} a_{1} T_{1}+c_{p} a_{2} T_{2}+c_{p} \delta a_{1}\left(T_{1}-T_{2}\right) \\
& =Q+c_{p} \delta a_{1}\left(T_{1}-T_{2}\right) .
\end{aligned}
$$

This is clearly false, since no heat has been added to the system and therefore $Q^{\prime}=Q$. For consistency, the new heats of regions 1 and 2 should be

$Q_{1}^{\prime}=c_{p} a_{1} T_{1}+c_{p} \delta a_{1} T_{2}$

$Q_{2}^{\prime}=c_{p}\left(a_{2}-\delta a_{1}\right) T_{2}$,

respectively. To account for this, the term added to the energy balance for region 1 must be $c_{p} a_{1} K_{T 1}=c_{p} \delta a_{1}\left(T_{1}-T_{2}\right)$ and that added to region 2 must be $K_{T 2}=0$ such that the intrinsic quantities $T_{1}$ and $T_{2}$ update by $K_{T_{1}}=\delta\left(a_{1}\right)\left(T_{1}-T_{2}\right) / a_{1}=\delta \ln \left(a_{1}\right)\left(T_{1}-T_{2}\right) \quad$ and $\quad K_{T_{2}}=0$, respectively.

Generalizing, a region with some intrinsic quantity $H_{i}$ (e.g., temperature, column atmospheric moisture per unit area, column soil moisture per unit area, column carbon dioxide per unit area, etc.) that expands into another region by an area $d a_{i}$, updates $H_{i}$ by $\delta a \delta H_{i j}$, where $\delta H_{i j}$ is the difference in quantities $H$ between the original region and the one it expanded into. A region that contracts, on the other hand, does not update $H$. For simplicity, we assume that every region except the accumulation zone, which is surrounded by an ablation zone, expands into and contracts against the region of bare land. This can be written as

$K_{H i}=\delta \ln \left(a_{i}\right)\left(H_{i}-H_{d}\right)$

for all such regions. The update for $H_{d}$ can be found from the conservation condition,

$K_{H d}=-\left[\sum_{i \neq d} a_{i} K_{H i}\right] / a_{d}$.

Specific regions in the model grow and contract according to their own rules. For instance, oceans and ice caps evolve according to water mass conservation, as described below. Vegetated areas, on the other hand, evolve according to the simple population dynamic rules

$\dot{a}_{i}=a_{i}\left(\beta\left(T_{i}\right) a_{d}-\gamma\right) \quad i=b, w$.

Here we are using

$\beta\left(T_{i}\right)=1-k\left(T_{i}-T_{\text {opt }}\right)^{2}$

as the birth rate of a species per unit area, with $T_{\text {opt }}$ the optimal temperature for both species' birth rate. The factor

$a_{d}=1-\sum_{i} a_{i}$

is the bare land fraction and $\gamma$ is the death rate for the species. This equation is known as the Lotka-Volterra equation (Boyce and DiPrima, 1992), a simple model of competition between two species for a single resource, space to grow.

Note that both the birth and death rates in (12) should arguably also be functions of some other local variables, such 


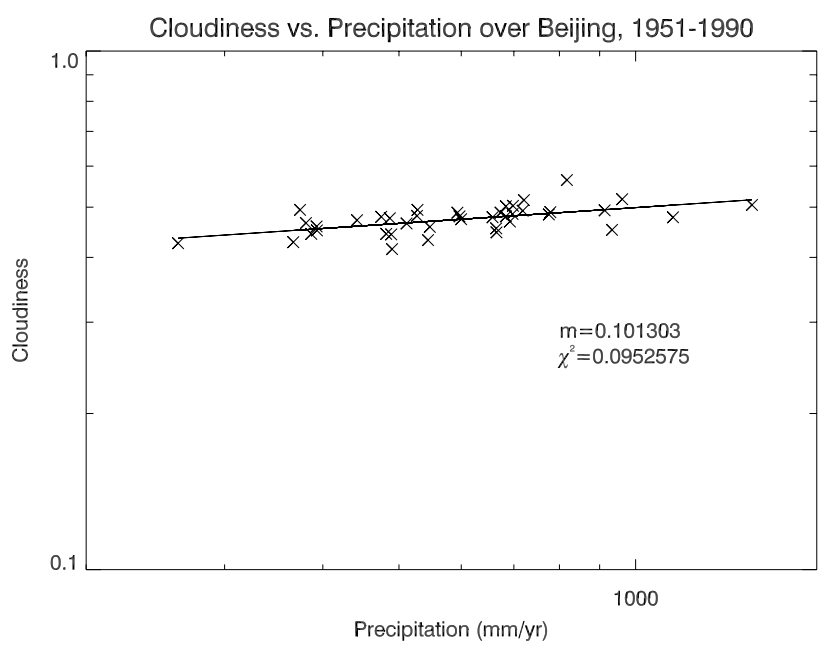

Fig. 1. Power law plot of rainfall and cloudiness over Beijing, China, 1951-1990. Data is from Wang et. al., 1993.

as soil moisture. However, because the size of the bare fraction $a_{d}$ is not determined solely by the biota, including other variables in the vegetation birth rate overdetermines the model. In such a case, trees and grasses may not simultaneously exist in steady state. We therefore elect to retain a birth rate dependent only on temperature.

\subsection{Hydrological cycle}

Water vapor is the largest greenhouse gas in the atmosphere. Therefore, the distribution of water in the atmosphere is important for specification of radiative effects like cloud reflectivity and greenhouse forcing. Functional relationships between surface water vapor characteristics and column integrated water vapor have been tested with some success (Choudhoury, 1996). We therefore choose to keep track of a given region's mean precipitable water, denoted $w_{i}$, as a simple measure of region mean atmospheric water vapor. Mass balance gives

$\dot{w}_{i}=E_{i}-P_{i}+F_{w i}+K_{w i}$

where $E_{i}$ is evaporation, $P_{i}$ is precipitation, $F_{w i}$ represents the interregional atmospheric moisture transport, and $K_{w i}$ is the DAF adjustment for precipitable water. We use the Magnus-Tetens formulae for estimating thermodynamic variables at saturation. Using the formula for saturation density $\rho_{S}(T)$ for water vapor, we can calculate the saturation mass per unit area for a vertical column of atmosphere using a linear temperature profile with lapse rate $\gamma_{l}=6.5 \mathrm{~K} / \mathrm{m}$ (Emanuel, 1994) with

$w_{\text {sat }}\left(T_{i}\right)=\int_{0}^{H} \rho_{s}\left(T_{i}+\gamma_{l} z\right) d z$.

A measure of the total relative humidity for a column can now be written $r_{i}=w_{i} / w_{\text {sat }}\left(T_{i}\right)$.

Precipitation $P_{i}$ is, of course, difficult to represent in a 0-D model. Convective rainfall is highly variable in both space and time, and to accurately represent this requires a very sophisticated parameterization. We elect, therefore, to simply view precipitation as the mechanism by which the atmosphere sheds excess water. As such, we leave a convection parameterization for the future and keep track of only annual mean rainfall, which has units of mass over time. The timescale should depend inversely upon local saturation, since a highly saturated atmosphere will rain more quickly than an unsaturated one. We therefore write

$P_{i}=f^{\prime} w_{i}=\left(f r_{i}^{p}\right) w_{i}$,

after the parameterization used by Paul (1996), which developed annual rainfall proportional to precipitable water, but with an additional power law dependence on column relative humidity. Mean annual regional cloudiness correlates logarithmically to precipitation (Fig. 1), so we take the regional cloud area fraction to be

$a_{c i}=a_{c o} P_{i}^{\alpha}$.

The empirical parameters $f, p, a_{c o}$, and $\alpha$ are to be determined from data.

Cloud albedo $A_{c i}$ is a sensitive function of cloud height (Hobbs and Deepak, 1981), and we assume that clouds form at a constant temperature $T_{c}$ across our model. A linear profile for temperature, which we have already assumed for saturation, means that $H_{c}\left(T_{i}\right)=\left(T_{i}-T_{c}\right) / \gamma_{l}$ and

$A_{c i}\left(T_{i}\right)=A_{c o}+\kappa H_{c}\left(T_{i}\right)$.

Here $T_{c}$ is the cloud top temperature from Eq. (3), $\frac{1}{\gamma_{l}}$ is the linear slope for cloud height, and $A_{c o}$ is the cloud albedo for clouds at the surface. The linear response of cloud albedo to cloud height, $\kappa$, is an empirical parameter to be determined from data.

To represent evaporation, we choose a Penman-Monteith resistance model of the form

$E_{i}=\frac{\left(R_{\text {in }, i}-R_{\text {out }, i}\right) \phi\left(T_{i}\right)+\rho_{a} c_{p a}\left(e_{s a t}\left(T_{i}\right)-e_{i}\right) r_{h}}{\left(\phi\left(T_{i}\right) r_{h}+\gamma_{h}\left(r_{h}+r_{s i}\right)\right) L_{v}}$,

where $\rho_{a}$ is the density of the ambient air, $c_{p a}$ is the heat capacity of the ambient air, $r_{h}$ is the hydrodynamic resistance of bare soil, $\gamma_{h}$ is the "psychrometer constant", and $L_{v}$ is the latent heat of vaporization of liquid water. $\phi\left(T_{i}\right)$ is an estimate of the derivative of the saturation vapor pressure $e_{s a t}\left(T_{i}\right)$ close to the ground (Monteith, 1981), $e_{i}=e_{o}\left[\ln \left(m_{w i}\right)+T_{c}\right]^{e_{x}}$ is the vapor pressure determined from the column integrated moisture (Emanuel, 1994), and $r_{s i}$ is the stomatal resistance of the region's biota. Stomatal resistance is a function of many things, most sensitively ambient carbon dioxide concentrations; but also intensity of sunlight, ambient temperature, and ambient relative humidity. Since carbon dioxide is fixed in this version of the model, in vegetated regions we take resistance to be a function of relative humidity

$r_{s i}=1 /\left(h_{1}+h_{2} r_{i}\right)$, 
setting $h_{1}=.004 \mathrm{~m} / \mathrm{s}$ and $h_{2}=.096 \mathrm{~m} / \mathrm{s}$ for consistency with the Ball-Berry model of stomatal conductance (Ball et al., 1987; Pleim, 1999). In non-vegetated regions, $r_{s i}=0$.

The greenhouse forcing, $v$, is taken to be a linear function of moisture. Here

$v=v_{c}+v_{w} m_{w i}$,

with $v_{c}$ the (constant) carbon forcing and $v_{w}$ the absorptivity of water across the visible and thermal spectra. Both parameters remain to be estimated from data.

Soil moisture is also an important variable to track, since its distribution determines, to a large extent, the ability of vegetation to survive. Mass balance for the soil gives us

$\rho_{w} d_{\text {soil }} \dot{s}_{w i}=\left(P_{i}-E_{i}+F_{s i}+K_{s i}\right)$,

for $s_{i}$ the soil water content in soil $d_{\text {soil }}$ deep; $\rho_{w}$ is the density of water. We choose $F_{s i}=D_{s}\left(\bar{s}-s_{i}\right)$, and $K_{s i}$ analogously to $K_{T i}$. Therefore, we take the death rate for biota to be a quadratic function of $s_{i}$, the simplest function that is zero at both saturation and drought.

We will assume for the purposes of this model that all interregional atmospheric transports occur through bulk movement of air, and thus through atmospheric circulation patterns. Then moisture transports in the atmosphere will follow the movement of heat, and they should reduce when there are smaller quantities of water in the atmosphere. We therefore make $F_{w i}$ proportional to $F_{T i}$ and the ratio of global mean precipitable water $\bar{w}$ to the mean precipitable water at the current luminosity, $\left.\bar{w}\right|_{L=1.0}$. This assumption yields

$F_{w i}=\left[D_{m}\left(\frac{\bar{w}}{\left.\bar{w}\right|_{L=1.0}}\right)\right] F_{T i}$,

in which $D_{m}$ is a constant determined by matching moisture content over the model ice caps to present day polar values with $\bar{w}=\left.\bar{w}\right|_{L=1.0}$. The value for $\left.\bar{w}\right|_{L=1.0}$ can be found from Peixoto and Oort (1992) to be about $25.5 \mathrm{~kg} / \mathrm{m}^{2}$.

\subsection{Oceans}

Oceans may be represented by a dynamic area fraction, with birth rate proportional to precipitation and runoff, and with death rate proportional to evaporation. Thus,

$\dot{a}_{o}=\left(a_{o}\left[P_{o}-E_{o}+F_{s o}\right]+a_{a b l} \mu_{i c e}\right) / M_{o}$

where $\mu_{i c e}$ is melt from the ablation zone of the polar caps and $M_{O}$ is the mass of a column of ocean water. We assume a constant mean ocean depth for simplicity. Although the actual adjustment of the ocean fraction is small due to the large value of the constant $M_{o}$, the effect of small changes in $a_{o}$ on the hydrological cycle in the rest of the model is potentially significant.

The presence of the soil moisture convergence term $F_{s o}$, included to capture the effects of runoff from land processes, requires the specification of a preferred soil moisture for the ocean, since we can't directly write down a sensible analog of soil moisture in the ocean. Then

$F_{\text {so }}=D_{s}\left(\bar{s}-s_{o}\right)$,

with $s_{O}$ a constant to be determined.

\subsection{Ice caps}

Ice caps can also be represented as a dynamic area fraction, but the DAF must be divided into two regions, an ablation zone and an accumulation zone. The division between these zones will be made by mean annual temperature, since annual snowmelt in the polar regions is principally determined by temperature and not by solar radiation (Bowman, 1982). We therefore construct a line of freezing $\theta_{f r z}$, inside of which ice sheets tend to grow and outside of which they ablate. The ice sheet poleward of $\theta_{f r z}$, where no significant snowmelt occurs, will be known as the "accumulation zone"; whereas the ice sheet towards the equator from $\theta_{f r z}$ will be referred to as the "ablation zone". This parameterization is constructed after the model discussed in Ghil and Childress (1987).

The freezing line is demarcated by a mean annual temperature equal to an effective freezing temperature, $T_{f e}$, discussed below. $\theta_{f r z}$ may then be determined from the global radiative balance by noting that the solar constant varies sinusoidally with co-latitude, and may be reasonably approximated by a square root for easy invertability. If we approximate the solar luminosity $\left.S_{\theta}=S\left(A_{\theta}+B_{\theta} \sqrt{(} \theta\right)\right)$, we can determine a co-latitude $\theta$ at which a given steady-state temperature $T_{\theta}$ will occur by using a steady state assumption on (1), such that

$\theta=\left[\left(R_{\text {in }}\left(w_{\theta}, T_{\theta}\right)+F_{T, \theta}\right) / R_{\text {out }}\left(w_{\theta}, T_{\theta}\right)-A_{\theta}\right]^{2} / B_{\theta}^{2}$.

Here $w_{\theta}$ is the local atmospheric moisture at temperature $T_{\theta}$, which can be found through a steady state assumption on a mass balance Eq. (14). Note that $K_{w, \theta}=0$ because $w_{\theta}$ is associated with a region of zero area.

The fractional area of a sphere that lies within a given co-latitude $\theta^{\prime}$ is $\left(1-\cos \theta^{\prime}\right) / 2$. If we assume north-south symmetry, this is multiplied by a factor of two such that the fractional area of a globe with an annual mean temperature lower than $T_{\theta}$ is $a_{\theta}=1-\cos \theta$. Hence, the accumulation zone has area $a_{a c c}=1-\cos \theta_{f r z}, \theta_{f r z}$ is the co-latitude at which snowmelt for the year is zero, and the ablation zone has area $a_{a b l}=a_{i}-a_{a c c}$.

Under the DAF formalism, the area of the ice sheet updates with different precipitation- and evaporation-dependent mass balance rates in each subregion so that

$a_{i}=a_{a c c}\left(P_{a c c}-E_{a c c}\right) / M_{I}+a_{a b l}\left(P_{a b l}-E_{a b l}-N_{a b l}\right) / M(2,7)$

where $N_{a b l}$ is a melt rate and $M_{I}$ is the mean mass of a column of ice. Note that this formulation assumes a square profile for the ice sheet for simplicity, though it is possible to assume other profiles, as well. Moisture and temperature variables must be calculated for each subregion to close $P_{a c c}$, $E_{a c c}, P_{a b l}$, and $E_{a b l}$. These variables are computed from their respective balances at the zonal center of each subregion.

DAF adjustment terms $K_{H i}$ become somewhat tricky over the ice. This is in part because the accumulation zone expands into the ablation zone rather than the bare land fraction 


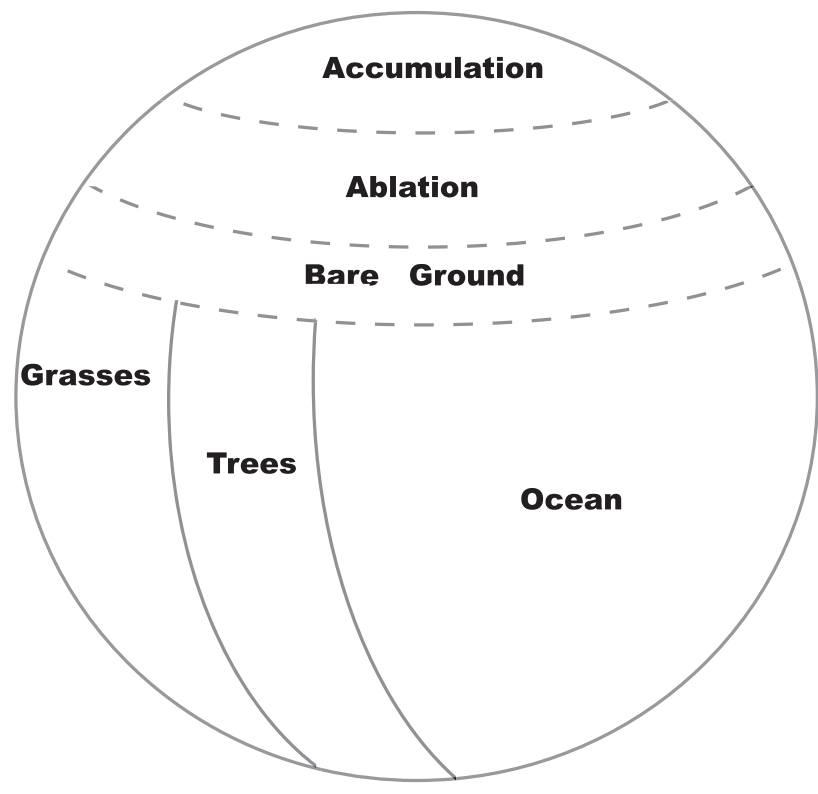

Fig. 2. Schematic of the layout of area fractions in this model. Ice caps are assumed to occur on the poles and to be symmetric. Dashed lines denote boundaries subject to dynamics, solid lines are fixed in time. Thus, all area fractions expand and contract into the bare ground region, except for the accumulation zone on the ice cap, which is surrounded by an ablation zone.

other regions expand into (see Fig. 2). Also, the ablation zone expands into both the accumulation zone and the bare land fraction. The fact that the ablation zone has two regions of expansion means it is necessary to specify in which direction the ablation zone has increased or decreased. Such a specification, though somewhat more complex than the DAFM behavior exhibited by the other regions, is not conceptually difficult. It simply relies on determining the growth of the ice sheet as a whole and $\theta_{f r z}$, then deductively specifying growth of the component parts. However, the analytical treatment is messy and not particularly illuminating physically, so we will not discuss it here, other than to note that the two-region expansion is accounted for in the model. This two-region expansion is the basis for developing a DAFM in higher dimensions, where one obtains a dynamically adjusting grid.

Over ice caps, snowmelt $N_{a b l}$ must also be computed. Energy balance models of the ice sheets (Bowman, 1982; Sandberg and Oerlemans, 1983; Paul, 1996) parameterize monthly snowmelt linearly in terms of monthly temperature $T_{m}$ as $N_{m}\left(T_{m}\right)=550+1100\left(T_{m}-T_{f}\right) \mathrm{kg} / \mathrm{yr}$ with $T_{f}$ the freezing point temperature $273.15 \mathrm{~K}$. $T_{m}$, of course, does not scale directly to an annual mean temperature, since the variation of monthly mean temperatures is substantial in the arctic regions. To reconcile this parameterization to an annual mean temperature, it is necessary to replace $T_{f}$ with an effective annual freezing temperature, $T_{f e}$ such that

$N_{a b l}=550+1100\left(T_{a b l}-T_{f e}\right) \mathrm{kg} / \mathrm{m}^{2} \mathrm{yr}$.
$T_{f e}$ is tuned to yield an ice-cap coverage of $2.9 \%$ since the major land ice sheets on earth, Greenland and Antarctica, cover approximately that much of earth's surface area.

\section{Feedbacks}

A model of this level of complexity contains many nonlinear processes, and as such it may be useful to enumerate feedbacks that have been included. For this purpose, we rewrite Eqs. (1) and (14) as

$$
\begin{aligned}
c_{p} \dot{T}_{i}= & S L A_{i}^{\prime}+\sigma v_{i} T_{i}^{4}+\sigma a_{c i}\left[\left(1-v_{i}\right) T_{i}^{4}-T_{c}^{4}\right] \\
& -S L A_{i}^{\prime} a_{c i} A_{c i}-\sigma T_{i}^{4} \\
& +F_{T i}+K_{T i}
\end{aligned}
$$

the expanded local heat update equation, and

$\dot{w}_{i}=E_{i}\left(w_{i}, T_{i}\right)-f w_{i}^{(1+p)} w_{s a t, i}\left(T_{i}\right)^{-p}+F_{w i}+K_{w i}(30)$

the equation for local moisture update with precipitation expanded. The effect of each term in these equations is more clear when expanded, since positive feedbacks are represented by those terms added and negative ones are represented by those subtracted.

The most obvious feedback is that between temperature and the blackbody term, the 5th term in (29), since it is uncoupled to other model variables. As local temperature $T_{i}$ increases, the amount of longwave radiation released from the atmosphere increases, evidently as $T_{i}^{4}$. This powerful negative feedback provides a temperature bound for the climate system.

However, an increase in a local temperature also leads to increases in several diagnostic model variables. Both evaporation $E_{i}$ (19) and precipitation $P_{i}(16)$ are increased, as is seen in more complex models (Houghton et al., 2005 ${ }^{1}$ ). Since $m_{s a t, i}$ in the denominator of $P_{i}$ is exponential in temperature, the saturation point of the atmosphere increases, so $m_{w i}$ increases as well.

The greyness factor $v_{i}$ (21) therefore increases, as does cloud area as a function of the precipitation and cloud albedo as a direct function of temperature (see Eqs. 17 and 18). $v_{i}$ appears in (29) coupled to temperature in the 2 nd term, creating a powerful positive feedback well known as the "Greenhouse" feedback.

Cloud fraction $a_{c i}$ appears in the 3rd and 4th terms of (29). Its effect in the 4th term is clearly that of a negative feedback, since it is coupled to the cloud albedo which also increases with temperature. This is commonly known as the shortwave cloud feedback. The effect of clouds on the 3rd term is more complex; for values of the cloud top temperature

$T_{c}<\left(1-v_{i}\right)^{1 / 4} T_{i}$

\footnotetext{
${ }^{1}$ Houghton, J., Filho, L. M., Callender, B., Harris, N., Kattenberg, A., and Maskell, K.: IPCC Third Assessment, in draft form, 2005.
} 
Table 1. Major feedbacks included in the full model.

\begin{tabular}{lcl}
\hline Feedback & Effect & Components involved \\
\hline blackbody & negative & temperature, heat \\
cloud albedo & negative & atmospheric moisture, temperature \\
biota albedo & negative & plant fraction, temperature \\
greenhouse & positive & atmospheric moisture,temperature \\
ice albedo & positive & ice fraction,temperature \\
cloud longwave & positive $T_{c}<\left(1-v_{i}\right)^{1 / 4} T_{i} ;$ else, negative & atmospheric moisture, temperature \\
\hline
\end{tabular}

this feedback will be positive, but for $T_{c}>\left(1-v_{i}\right)^{1 / 4} T_{i}$ this feedback is negative! That is, for high values of the greyness factor in this model, the effect of clouds in the longwave can actually be to cool the planet, an interesting feedback to be sure. However, it must be noted that such a situation is not physically realistic, since heat must first escape from the greenhouse gases below the clouds in order to reach the clouds and the model does not account for this. The possibility of such a feedback is thus an artifact of our assumption of a constant cloud top temperature. We can view relation (31) as providing an upper bound for estimation of the parameter $T_{c}$ in the model.

There is an ice-albedo feedback in the model, a positive feedback produced by the high reflective capabilities of polar ice. The accumulation angle $\theta$ (26) increases as a function of $F_{T, \theta}$, which increases as $T$ decreases. Thus colder temperatures lead to a larger accumulation of ice, which reflects more heat.

Other feedbacks come from the surface processes in the model. From Eq. (11) with $\beta\left(T_{i}\right)$ expanded and $\gamma\left(s_{i}\right)=\gamma_{o}+$ $\gamma^{\prime}\left(s_{i}-s_{\text {opt }}\right)^{2}$,

$\dot{a}_{i}=a_{i} a_{d}-a_{i} a_{d} k\left(T_{i}-T_{o p t}\right)^{2}-a_{i} \gamma$

The second term in this equation is a biota albedo feedback, negative since it increases as the local temperature strays from the optimal.

Feedbacks with ocean fraction are negligible, since the percent change of the area of the ocean system is extremely small. Major feedbacks in the model are listed in Table 1.

\section{Parameter Estimation}

As with any model, the DAFM requires the specification of several parameters and we choose these to resemble an earthlike climate. As a general and consistent rule, model parameters are selected to bring the system variables to approximately match annual means on earth. However, the parameterizations themselves are applied by linearly scaling the annual mean estimates to the time scale of model integration, $1 / 1000$ th of a year, and by varying them on that timescale.

Our representation of the hydrological cycle demands specification of a fallout frequency, $f$; the relative humidity exponent $p$ for precipitation; a base cloudiness, $a_{c o}$; the precipitation exponent for cloudiness, $\alpha$; the surface temperature slope for cloud albedo, $\kappa$; the minimum albedo for clouds, $A_{c o}$; the greenhouse greyness due to carbon, $v_{c}$; the water vapor slope for water vapor greyness, $v_{w}$; the preferred oceanic soil moisture, $s_{o}$; the coefficient for the linear transport of atmospheric moisture, $D_{w}$; the coefficient for the linear transport of soil moisture, $D_{s}$; the snowmelt temperature, $T_{f e} ;$ and the cloud top temperature $T_{c}$.

We use data taken over Beijing, China (Wang et al., 1993) to find our power law exponent, $\alpha=.1$, indicating a weak dependence for cloudiness on precipitation (Fig. 1). Since the earth's mean cloudiness at our current temperature is $49 \%$, we can find $a_{c o}$ from the mean conditions. The mean surface temperature of the earth is around $288.4 \mathrm{~K}$, its mean precipitable water is about $25.5 \mathrm{~kg} / \mathrm{m}^{2}$, and its mean albedo is 30 $35 \%$ (Peixoto and Oort, 1992). The minimum cloud albedo $A_{c o}$, generally associated with low-lying fog, we take to be .05 to match the albedo of the ocean. For stability the model requires $\kappa \leq 1 / 60$, and for larger $\kappa$ the clouds become increasingly more capable of adjusting albedo to block solar input; thus we take $\kappa$ to be its minimum possible value to prevent excessive bias towards homeostatic behavior in the hydrological cycle. Then, from the earth's mean albedo, we can determine the cloud top temperature $T_{c}$.

Ice sheets cover $2.9 \%$ of the earth's surface, which allows us to determine the annual melting temperature $T_{f e}$. The precipitation exponent $p$, representing the power law dependence of precipitation frequency on column humidity, we determine by matching the frequency found from data in the ice cap model of Paul (1996) at earth-like conditions in our parameterization. We can now determine the frequency coefficient $f$ from the mean column moisture on earth.

The moisture transport coefficient we assume to be constant over the globe by construction; its value can be estimated from the polar moisture transport listed in Peixoto and Oort (1992). For simplicity, we use $D_{w}=D_{s}$, dividing moisture flux evenly between atmosphere and land. Model behavior is relatively insensitive to variations in this parameter in the neighborhood of its estimated value.

The carbon greyness $v_{c}$ is tuned to represent about $5 \%$ of the greenhouse forcing. For a soil composed of $10 \%$ sand and $10 \%$ clay, saturation occurs at $s_{i} \approx .45$ and drought for $s_{i} \approx .1$ (Salisbury and Ross, 1992). The ocean's preferred soil moisture, $s_{o}$, is set to the midpoint of these two values, $s_{o}=.275$. 


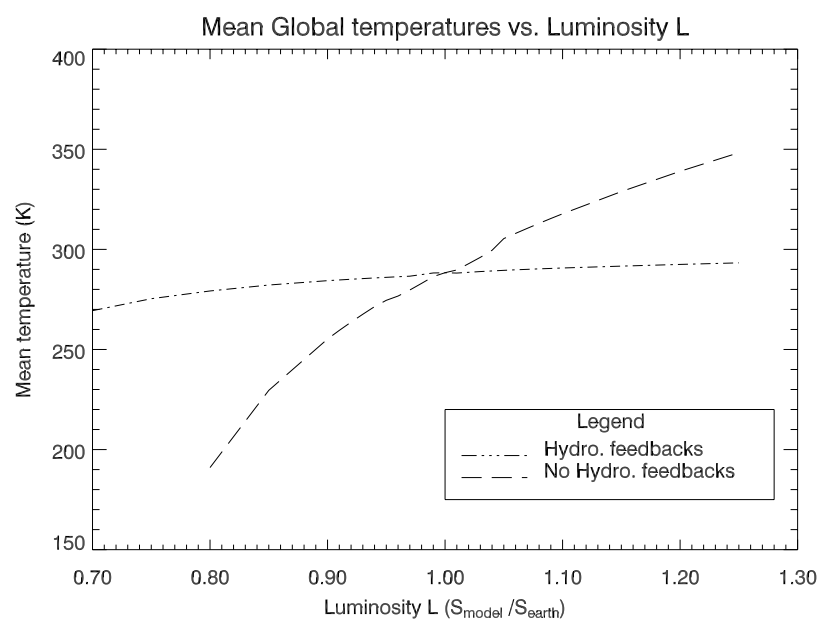

Fig. 3. Comparison of global mean temperature $T$ vs. luminosity $L$ in a DAFM integration with fixed hydrological cycle to a DAFM with a dynamic hydrological cycle. Biota is dynamic and identically forced in both plots. The hydrological cycle in the full DAFM produces an exceptionally strong stabilizing effect.

Taking a conservative estimate of the carbon greenhouse greyness, we can determine $v_{w}$ finally from the earth's mean temperature. Values of parameters used in our model runs for this analysis are listed in Tables 2 and 3.

\section{Analysis}

We perform two numerical experiments to emphasize the effects of feedbacks between various components of the hydrological cycle on the global mean temperature of the model. In the first experiment, we compare runs of the fully coupled model with runs of a reduced model implementing only static hydrological variables over a range of solar luminosities $L$.

In the second experiment, we modify the $T_{o p t}$ parameter, the uniform optimal temperature for seedling growth of both trees and grasses in the model. In the real ECS, this parameter may vary from species to species, and is not well known in general, so modifying the parameter serves two purposes: 1., to test model sensitivity to an unknown value; and 2., to show the range of control vegetation can have over surface temperatures through changing evaporative properties and albedo.

As a basis for comparison in both experiments, we run the model to a steady state for earthlike conditions, as defined by a modern solar luminosity $(L=1)$, a mean temperature near $288.5 \mathrm{~K}$, mean precipitable water near $25.5 \mathrm{~K}$, an ice cap fraction near 3\%, cloudiness near 50\%, realistic temperatures and precipitation near the poles, and approximately equal coverage for our two plant species.

\subsection{Comparison to a fixed hydrological cycle varying $L$}

The intent of this experiment is to establish a qualitative idea of the combined effects of various feedbacks between the hydrological cycle and the heat balance on a planet like earth.
As such, we first compile data from a set of runs of the full model with solar luminosities ranging from $70 \%$ of present day insolation to $130 \%$. In what follows, we shall refer to this set with the label $\epsilon(L)$.

For the second set of runs, labelled $\Phi(L)$, we fix all variables associated with the hydrological cycle in the model, including regional cloudiness, regional cloud albedo, ice fraction, regional precipitable water, ocean fraction, regional precipitation, and regional evaporation, to the values obtained in the earthlike run, $\epsilon(L=1)$. Like $\epsilon(L)$, this data set is obtained by varying $L$. Vegetation fractions and energy balances are the only climate components in $\Phi(L)$ allowed to respond to changes in insolation.

The contrast between the surface temperature values in the full model and those in that without water feedbacks is striking (Fig. 3). Changes in the global mean temperature in $\epsilon(L)$ are substantially smaller than they are in $\Phi(L)$, indicating that an active hydrological cycle represents a tremendous net negative feedback for all values of $L$. This result is particularly interesting in light of the fact that two very strong and extremely important positive hydrological feedbacks are present in $\epsilon(L)$ but not in $\Phi(L)$, namely the ice-albedo and hydrological greenhouse feedbacks.

Since these are well-known to be positive feedbacks, other hydrological quantities in the radiative balance must be responsible for this behavior. The only ones remaining are the nonphysical longwave cloudiness effect and the shortwave cloud albedo effect, which represent two possible mechanisms for a stabilizing effect. The first is due to an increase of both cloud area and cloud albedo in the shortwave term with increasing temperatures, and the second is due to the increased absorption and reemission of longwave radiation from the surface in the presence of more clouds. We would like to show that the latter is not the cause of this behavior, since we have discussed earlier that it is not physically relevant.

In Fig. 4 we plot the incoming shortwave radiation at the model's surface over the five regional surface types. The important feature in this plot is that the surfacial insolation on three of the four upper regional curves vary from their means on the order of $5 \%$ or less over the entire range of values of $L$ tested. The only exceptions are the region of bare ground, which varies on the order of $7 \%$, and the ice cap, which covers a very small fraction of the surface. This range of $L$ represents a variation of $30 \%$ from its mean, so the behavior of the shortwave to solar forcing is extremely stable. It should also be noted that the slopes of these lines are generally decreasing with $L$. From Eq. (1) and the fact that the model resides at steady state, we can loosely approximate this behavior as a constant. Then we can write

$R_{\text {in,i }}=R_{\text {out }, i}-F_{T i}$,

in which all temperature dependence resides on the right side and the left side is approximately a regionally dependent constant, $R_{i}$. Summing over regions to eliminate the transport 
Table 2. Parameters used in DAFM model runs.

\begin{tabular}{llll}
\hline parameter & value & meaning & source \\
\hline$S$ & $1.07 \mathrm{e} 10 \mathrm{~J} / \mathrm{m}^{2} \mathrm{yr}$ & incident solar output & Peixoto and Oort (1992) \\
$s_{f 1}$ & .5 & lat. temp. profile intercept & Peixoto and Oort (1992) \\
$s_{f 2}$ & .6 & l. temp slope w/ $\sqrt{\theta}$ & Peixoto and Oort (1992) \\
$c_{p l}$ & $1.8 \mathrm{e} 9 \mathrm{~J} / \mathrm{K} \mathrm{m}^{2}$ & earth heat capacity & calculated \\
$D_{w}$ & $1000 . \mathrm{kg} / \mathrm{m}^{2} / \mathrm{yr}$ & moisture transport (atmo) & Peixoto and Oort (1992) from polar flux at 70N \\
$B_{I}$ & $550 . \mathrm{kg} / \mathrm{yr}$ & base melting rate & Bowman (1982) \\
$\rho_{w}$ & $1000 \mathrm{~kg} / \mathrm{m}^{3}$ & density of liquid water & Peixoto and Oort (1992) \\
$d_{s}$ & $1 \mathrm{~m}$ & soil depth & avg. root depth Salisbury and Ross (1992) \\
$v_{c}$ & .217 & carbon greyness contribution & arbitrary, less than water \\
$v_{w}$ & .0157 & water greyness contribution per kg & tuned for mean temperature \\
$W s l o p e$ & 1.6 & dewpoint slope with atm moisture & Hobbs and Deepak (1981) \\
$S_{A c}$ & $60 \mathrm{~m}-1$ & cloud albedo slope with height & set for stability \\
$A_{c o}$ & .05 & base cloud albedo & set to match oceans \\
$A_{b}^{\prime}$ & .85 & tree co-albedo & McGuffie and Henderson-Sellers (1997) \\
$A_{w}^{\prime}$ & .75 & grass co-albedo & McGuffie and Henderson-Sellers (1997) \\
$A_{d}^{\prime}$ & .8 & bare land co-albedo & McGuffie and Henderson-Sellers (1997) \\
$A_{o}^{\prime}$ & .9 & ocean co-albedo & McGuffie and Henderson-Sellers (1997) \\
$A_{a c c}^{\prime}$ & .2 & accumulation co-albedo & McGuffie and Henderson-Sellers (1997) \\
$A_{a b l}^{\prime}$ & .6 & ablation co-albedo & McGuffie and Henderson-Sellers (1997) \\
\hline & & & \\
\hline
\end{tabular}

Table 3. Parameters used in DAFM model runs, cont'd.

\begin{tabular}{llll}
\hline parameter & value & meaning & source \\
\hline$D_{T}$ & $1.3 \mathrm{e} 8 \mathrm{~J} / \mathrm{m}^{2} \mathrm{yr}$ & heat transport (atmo) & Budyko (1969) \\
$k$ & .003625 & plant death exponent & Watson and Lovelock (1983) \\
lapse & .0065 & atmos. temperature lapse rate & Peixoto and Oort (1992) \\
$\sigma$ & $1.79 \mathrm{~J} / \mathrm{K}^{4} \mathrm{yr} \mathrm{m}^{2}$ & Stefan-Boltzmann blackbody const & Peixoto and Oort (1992) \\
$T$ opt & $286.23 \mathrm{~K}$ & optimal growth temperature & set for even occupation \\
$T_{f e}$ & $269.3 \mathrm{~K}$ & annual temp for ice accumulation & set to match ice fraction \\
$\alpha$ & .1 & rainfall-cloudiness exponent & Wang et al. (1993) \\
$c_{a}$ & $1006 \mathrm{~J} / \mathrm{K} \mathrm{kg}$ & heat capacity of air & Morton (1983) \\
$h_{1}$ & 10 & base hydrodynamic resistance & set - small range of res. \\
$h_{2}$ & 90 & hyd. res. slope with humidity & (from 10 yr/m ${ }^{2}$ to 100) \\
$L_{f}$ & $.3337 e 6$ & latent heat of freezing (water) & Peixoto and Oort (1992) \\
$L_{S}$ & $2.834 e 6$ & latent heat of sublimation & Peixoto and Oort (1992) \\
$L_{v}$ & $2.46 e 6$ & latent heat of vaporization & Peixoto and Oort (1992) \\
$m_{i c e}$ & $560000 \mathrm{~kg} / \mathrm{m}^{2}$ & column mass of ice & Peixoto and Oort (1992) \\
$m_{\text {oo }}$ & $3800000 \mathrm{~kg} / \mathrm{m}^{2}$ & column mass of ocean & Peixoto and Oort (1992) \\
prexp & .1 & exponent for precip. with hum. & Paul (1996) \\
$\pi_{c}$ & .68 & psychrometer constant & Morton (1983) \\
$r_{h}$ & $1.27 e-6 \mathrm{~s} / \mathrm{m}^{2}$ & resistance to sensible heat & Monteith (1981) \\
$\rho$ & $.87 \mathrm{~kg} / \mathrm{m}^{3}$ & density of air & Morton (1983) \\
$\rho_{i}$ & $800 \mathrm{~kg} / \mathrm{m}^{3}$ & density of ice & Peixoto and Oort (1992) \\
\hline & & &
\end{tabular}

terms,

$\sum_{i} a_{i} \sigma\left(1-a_{c i}\right)\left(1-v_{i}\right) T_{i}^{4}=\sum_{i}\left[R_{i}-\sigma a_{i} a_{c i} T_{c}^{4}\right]$

If $a_{c i}=\bar{a}_{c}+\epsilon_{i}, v_{i}=\bar{v}+\delta_{i}$, and $T_{i}=\bar{T}+\tau_{i}$, then we can approximate the above as

$$
\begin{gathered}
\sigma\left(1-\bar{a}_{c}\right)(1-\bar{v}) \bar{T}^{4}\left[1-\frac{\sum_{i} a_{i} \epsilon_{i}}{1-\bar{a}_{c}}-\frac{\sum_{i} a_{i} \delta_{i}}{1-\bar{v}}+\frac{4 \sum_{i} a_{i} \tau_{i}}{\bar{T}}\right] \\
\approx \sum_{i}\left[R_{i}-\sigma a_{i} a_{c i} T_{c}^{4}\right]
\end{gathered}
$$

whence it is a simple matter to show that the three sums in the brackets are small. Thus, with $\bar{R}=\sum_{i} a_{i} R_{i}$,

$$
\begin{aligned}
\bar{T}^{4} & \approx\left[\bar{R}-\sigma \bar{a}_{c} T_{c}^{4}\right] / \sigma\left(1-\bar{a}_{c}\right)(1-\bar{v}) \\
& =\frac{\bar{R}}{\sigma}\left[\frac{1-\bar{a}_{c}\left(\sigma T_{c}^{4} / \bar{R}\right)}{1-\bar{a}_{c}}\right]\left[\frac{1}{1-\bar{v}}\right] .
\end{aligned}
$$

The first factor in this equation expresses all shortwave effects in the model, the second expresses the possible 


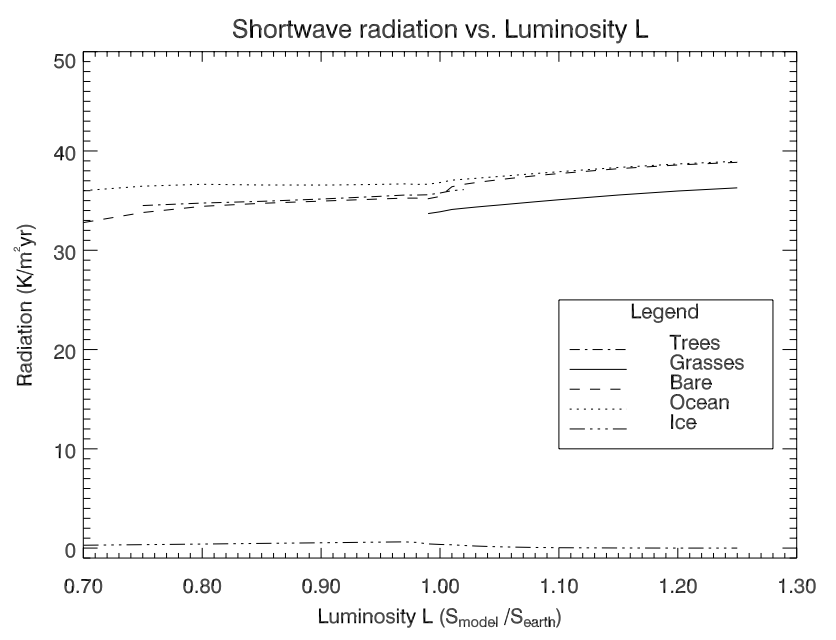

Fig. 4. Shortwave radiation at the surface by region for model runs with fully coupled water, $\epsilon(L)$. Variations in the shortwave forcing that reaches the surface are typically very small, despite a substantial change in the insolation at the top of the atmosphere.

non-physical negative feedback between cloud fraction and heat balance in the longwave (note that it is both physical and positive if condition (31) is met), and the third expresses the positive feedback inherent in the greenhouse effect.

We can now contrast the behavior of this equation in $\epsilon(L)$ and $\Phi(L)$ to see that it is the behavior of clouds in the shortwave band, expressed by the first factor in (38), that most supports the stability. For the fixed-water set $\Phi(L)$, neither $\bar{a}_{c}$ nor $\bar{v}$ change at all; all changes in $\bar{T}^{4}$, therefore, are caused by changes in $\bar{R}$, which is linearly dependent on $L$. For $\epsilon(L)$, on the other hand, $\bar{R}$ is nearly fixed, as shown in Fig. 4. $\bar{a}_{c}$ increases with increasing temperature for $\epsilon(L)$, and since $\sigma T_{c}^{4} / \bar{R}<1$, the second factor in (38) is also increasing with increasing temperature (though it is slowed by the longwave negative feedback with cloud fraction). $\bar{v}$ increases fairly rapidly, and so the third factor in (38) is the source of the most warming in $\epsilon(L)$. Thus, the second two factors in $\epsilon(L)$ drive the warming; and indeed, warm the model more than their counterparts in $\Phi(L)$ (which do not increase at all). Since the first factor increases very slowly for $\epsilon(L)$, it is clearly the source of the extraordinary stability.

\subsection{The Effect of varying $T_{\text {opt }}$ on surface temperatures}

The $T_{\text {opt }}$ parameter is difficult to estimate from data, especially considering that it probably varies relatively widely over species adapted to different climatic zones. However, it plays a relatively critical role, as it determines the range of temperatures over which the two species state (simultaneous trees and grasses) can exist.

In our DAFM, $T_{o p t}$ is taken to be uniform among trees and grasses, for simplicity. For the first experiments above, it was tuned to allow both species to occupy equal area fractions. Determining the sensitivity of our model to this parameter gives not only an idea of the range of error associated in quantitatively applying results from our model to earth, but also a way to demonstrate the full extent of the biota's control over its environment.

We vary $T_{o p t}$ over a wide range of temperatures on the interval $[260,310] \mathrm{K}$ and run the DAFM at earthlike conditions, using the $\epsilon(L=1)$ run from the previous test as initial conditions. The results are shown in Fig. 5. The plot breaks into five regions: one two-species region, two single-species regions, and two regions without biota. The two "dead" regions, on the intervals $\bar{T} \in[260,271] \mathrm{K}$ and $\bar{T} \in[302,310] \mathrm{K}$, are at the same fixed temperature representing the global mean dominated by ocean and desert.

The three "live" regions may be characterized by species dominance. The left hand region, in which the mean temperature $\bar{T}$ is warmer than $T_{o p t}$, is dominated by the high albedo grasses. Here $\bar{T}$ is steadily reduced with increasing $T_{\text {opt }}$ as grasses become capable of covering a greater fraction of the surface. The right hand region is dominated by the low-albedo trees, since $\bar{T}$ is cooler than $T_{o p t}$. In this region, $\bar{T}$ also reduces with increasing $T_{o p t}$ as trees are increasingly less capable of covering the surface. The central region of the plot, in which both species share dominance of the planet, shows high sensitivity to $T_{o p t}$, as competition between trees and grasses adjusts the global mean temperature by more than $1 \mathrm{~K}$. This suggests a surprising degree of control over the mean planetary temperature, as does the fact that the existence of trees and grasses in various configurations appears capable of adjusting $\bar{T}$ by about $1.6 \mathrm{~K}$.

\section{Conclusion}

With our simple hydrological DAFM, we have performed two numerical experiments to investigate the effects of coupling the hydrosphere and the biosphere on the global mean temperature of an earth-like planet.

In the first, we heated the sun and observed a spectacular negative feedback reaction, as the surface heating required the presence of more and more clouds to produce precipitation. While it is possible that this behavior is larger than it should be on earth, it should be noted that the mechanism itself is more than plausible in the ECS. The presence of water in large amounts such as are found on our planet indicates that the heat capacity should be large, a fact that immediately dictates a slow response time for changes in the radiative balance. Increased surface heating of the earth will thus more rapidly produce changes in evaporation, in turn requiring increased precipitation and therefore cloudiness. This is a direct result of the fact that the atmospheric capacity to hold water vapor is a monotonic function of temperature, and is qualitatively unaffected by parameterization. Quantitatively it is, of course, as we have used a primitive linear estimation of greenhouse dependence that ignores saturation effects and therefore overestimates the strength of this positive feedback.

The radiative properties of clouds themselves is a subject for debate, since they apparently include two opposing mechanisms. Clouds generally have high albedos when compared 

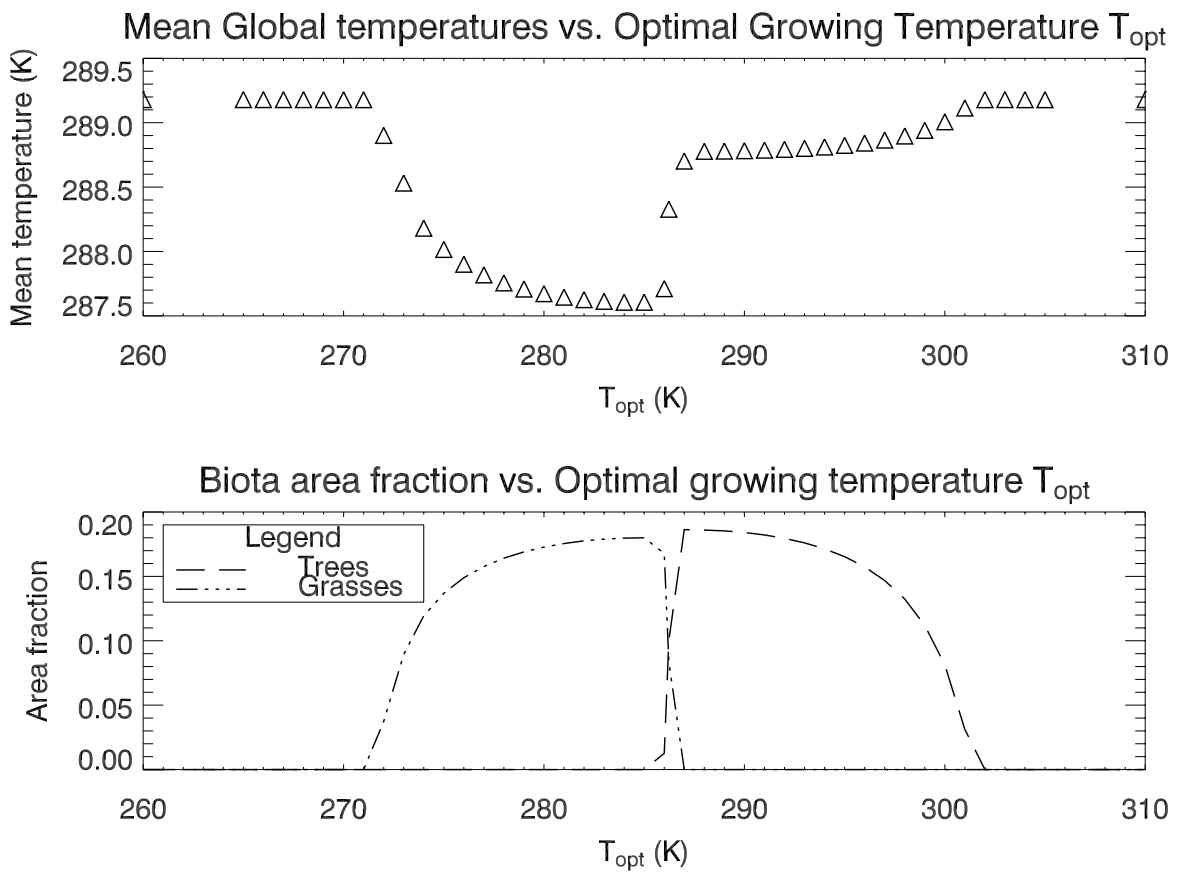

Fig. 5. The effect of varying the optimal growth temperature $T_{\text {opt }}$ on the global mean temperature $T$. The temperature to the left and right of the upper plot is the temperature of a dead planet with no biota. Rapid changes in the center of the plot are due to nonlinear competition as the two species (trees and grasses) respond sensitively to changes in $T_{\text {opt }}$.

to the surface of the earth, and so their effect on the shortwave radiative term is to reflect away a greater quantity of the sun's input. This, of course, must be viewed as a cooling effect. Clouds are also efficient absorbers of longwave radiation and form at low temperatures such that the blackbody radiation they produce is much lower than that produced at the ground. This latter effect dictates that clouds also create a warming effect in the longwave term. While hardly conclusive, the results from our model indicate that this effect is dominated by the relative constancy of the shortwave term, a behavior associated with the cloud albedo feedback.

It is possible that our choice for the cloud albedo slope $\kappa$ has affected the qualitative results of this experiment. We have used the minimum value available to us in the present configuration to reduce such an effect. However, we will test this possibility in a future paper. Our parameterizations for the longwave terms that could have affected results include our representation of the greenhouse effect; our assumption of fixed cloud top temperatures $T_{c}$; and our choice, determined as it was by other model parameters and variables, for the cloud top temperature $T_{c}=270 \mathrm{~K}$. An increased carbon greenhouse effect $v_{c}$ relative to water vapor would lower the cloud longwave term, while leaving the ground longwave term approximately the same; this change would also not qualitatively affect results, since in this model carbon is treated as a static parameter. Changing cloud top temperatures $T_{c}$ would have exactly the same effect.

It is possible that removing the assumption of constant cloud top temperatures in our model could qualitatively change the outcome of our model runs, and it is possible that allowing carbon to dynamically adjust to climatic changes could do so as well. However, such a situation would require very strong positive feedbacks in the carbon cycle or the cloud top temperatures. In a future paper, we intend to examine results from runs with both of these assumptions removed.

It is interesting to note that these model runs produced no "iceball" effect, even at tremendously low luminosities $L$. We have also tested much lower values of the heat transport parameter $D_{T}$ and found only modest changes in the size of the ice sheet. This phenomenon is even more interesting in light of the fact that we used the linear Budyko parameterizaton for heat transport, which was cited as a cause of the iceball effect in his seminal paper (Budyko, 1969; Sellers, 1969). It is possible that the presence of the feedbacks in our model's active hydrological cycle or its biota has prevented this from occurring. However, at present such a conclusion would be speculative, since we have not tested the system sufficiently thoroughly to determine why the ice caps do not grow to cover the system.

In the second experiment, we changed growing characteristics associated with the biota by adjusting the optimal temperature for seedling growth, $T_{\text {opt }}$. We found that the range of temperature difference exerted by adjusting the biota fractions was around $1.6 \mathrm{~K}$, a change on the same order of magnitude as that predicted by GCMs for the doubling of $\mathrm{CO}_{2}$ in Earth's atmosphere (Peixoto and Oort, 1992). This is a significant result, since there are more than enough components in this model to dilute the effects of the biota. 
It remains to add dynamic carbon feedbacks to this model, like those studied in Svirezhev and von Bloh (1998). A future paper coupling the two cycles would certainly produce interesting results. It would likewise be interesting to establish local heat capacities, $c_{p i}$. In addition, more spatial dependency should be added, as biota have different growing characteristics at different latitudes and the effect of landform location is suspected of playing some role in determining global climate. Using different optimal growing temperatures for trees and grasses might also allow us to include growth dependence on other model variables, such as soil moisture.

More generally, there is a great need in this and other models to develop a rigorous framework for parameterizations, one that explicitly incorporates the basic issue of scale dependence and scale invariance in biophysical processes. For example, in this model and others (Pan, 1990), a PenmanMonteith equation has been used to represent evapotranspiration at relatively large spatial scales. However, Choudury (1999) found that the Penman-Monteith equation was not scale invariant across a very broad range of spatial scales tested in the biophysical model they studied. Such a result reinforces that the Penman-Monteith equation is valid only for very specific scales and should not be directly applied to larger ones. At present, the study of scaling transformations on biophysical parameterizations is in its infancy (e.g. Milne et al. (2002)). Thus the biophysical parameterizations available to modelers at present are typically not scaleappropriate.

Finally, there is a need to establish model parameterizations in all climate models consistent with some established set of basic physical principles. Unfortunately, there has been relatively little attention paid to how to elucidate such a set of principles in a system like Earth's climate system, which is a highly nonlinear system far from equilibrium or steady state. For example, a promising candidate in this area is the Maximal Entropy Production (MEP) formalism developed by Paltridge (1975) and others (for an excellent review see Ozawa et al. (2003)). Only by establishing such a consistent theoretical framework will we be able to effectively develop parameterizations capable of representing higher-order moments as well as the means, or ensure consistency in our physical schemes and the results. In future DAFM models we intend to explore the development of parameterizations under a MEP formalism.

Acknowledgements. The first author would like to thank the National Science Foundation (NSF) for its generous support under a Graduate Research Traineeship (GRT) grant awarded to the University of Colorado hydrology program, which made the development of this work possible as part of his $\mathrm{PhD}$ dissertation. He and the second author would also like to acknowledge NSF for its continued support as the work evolved under grants EAR-9903125 and EAR-0233676.The third author wishes to acknowledge NSF grant number ATM-0001476 in support of this research. All the three authors gratefully acknowledge the CIRES Innovative Research Program for its support of this research.
Edited by: B. Sivakumar

Reviewed by: two referees

\section{References}

Ball, J., Woodrow, I., and Berry, J.: A model predicting stomatal conductance and its contribution to the control of photosynthesis under different environmental conditions, Progress in Photosynthesis Research, IV, 221-234, 1987.

Bowman, K.: Sensitivity of an annual mean diffusive energy balance model with an ice sheet, J. Geophys. Res., 87, 9667-9674, 1982.

Boyce, W. and DiPrima, R.: Elementary differential equations and boundary value problems, 5th ed., Wiley, New York, 1992.

Budyko, M.: The effect of solar radiation variations on the climate of the Earth, Tellus, 5(9), 611-619, 1969.

Choudhoury, B.: Comparison of two models relating precipitable water to surface humidity using globally distributed radiosonde data over land surfaces, Int. J. Climatol., 16, 663-675, 1996.

Choudury, B.: Evaluation of an empirical equation for annual evaporation using field observations and results from a biophysical model, J. Hydrol., 216, 99-110, 1999.

Claussen, M., Crucifix, M., Fichefet, T., Ganopolski, A., Goosse, H., Lohmann, G., Loutre, M.-F., Lunkeit, F., Mohkov, I., Mysak, L., Petoukhov, V., Stocker, T., Stone, P., Wang, Z., Weaver, A., and Weber, S.: Earth system models of intermediate complexity: Closing the gap in the spectrum of climate system models, Clim. Dyn., 18, 579-586, 2002.

Emanuel, K.: Atmospheric convection., Oxford Univ. Press, New York, 1994.

Ghil, M. and Childress, S.: Topics in geophysical fluid dynamics: Atmospheric dynamics, dynamo theory, and climate dynamics, Springer-Verlag, NY, 1987.

Harvey, L. and Schneider, S.: Sensitivity of internally generated climate oscillations to ocean model formulation, in: Proceedings on the Symposium on Milankovitch and Climate, edited by: Berger, A., Imbrie, J., Hays, J., Kukla, S., and Saltzman, B., ASI, pp. 653-667, NATO, D. Reidel, Hingham, MA, 1987.

Hobbs, P. and Deepak, A.: Clouds: Their formation, optical properties, and effects, Academic Press, NY, 1981.

Houghton, J., Filho, L. M., Callender, B., Harris, N., Kattenberg, A., and Maskell, K.: Contribution of working group I to the second assessment of the intergovenmental panel on climate change, in: IPCC Second Assessment - Climate Change 1995, MIT Press, Cambridge, MA, 1995.

Lovelock, J.: Gaia as seen through the atmosphere, Atmos. Environ., 6, 579-580, 1972.

Lovelock, J. and Margulis, L.: Atmospheric homeostasis by and for the biosphere: the Gaia hypothesis, Tellus, 26, 1-10, 1974.

McGuffie, K. and Henderson-Sellers, A.: A climate modeling primer (Research and development in climate and climatology), J. Wiley and Sons, NY, 1997.

Milne, B., Gupta, V., and Restrepo, C.: A scale invariant coupling of plants, water, energy, and terrain, Ecoscience, 9(2), 191-199, 2002.

Monteith, J.: Evaporation and surface temperatures, Quarterly Journal of the Royal Meteorological Society, 107, 1-27, 1981.

Morton, F.: Operational estimates of areal evapotranspiration and their significance to the science and practice of Hydrology, J. Hydrol., 66, 1-76, 1983. 
Nordstrom, K.: Simple models for use in Hydroclimatology, Ph.D. thesis, University of Colorado at Boulder, 2002.

Nordstrom, K., Gupta, V., and Chase, T.: Salvaging the Daisyworld parable under the Dynamic Area Fraction Framework, in: Scientists Debate Gaia: the next century, edited by: Miller, J., Boston, P., Schneider, S., and Crist, E., MIT Press, Cambridge, MA, 2004.

O'Brian, D. and Stephens, G.: Entropy and climate II: Simple models, Quarterly Journal of the Royal Meteorological Society, 121, 1773-1796, 1995.

Ozawa, H., Ohmura, A., Lorenz, R., and Pujol, T.: The second law of thermodynamics and the global climate system: a review of the maximum entropy production principle, Rev. Geophys., 41(4), 1018, 2003.

Paltridge, G.: Global dynamics and climate - A system of minimum entropy exchange, Quart. J. Roy. Meteor. Soc., 101, 475-484, 1975.

Pan, H.-L.: A simple parameterization scheme of evapotranspiration over land for the NMC medium-range forecast model, Monthly Weather Review, 118, 2500-2512, 1990.

Paul, A.: A seasonal energy balance climate model for coupling to ice-sheet models, Ann. Glaciol., 23, 174-180, 1996.
Peixoto, J. and Oort, A.: Physics of climate, American Institue of Physics, NY, 1992.

Pleim, J.: Modeling stomatal response to atmospheric humidity, in Preprints of the 13th Symposium on Boundary Layers and Turbulence, January 10-15 1999 in Dallas, TX, American Meteorological Society, Boston,MA, 1999.

Salisbury, F. and Ross, C.: Plant physiology (4th ed.), Wadsworth Publishing Co., Belmont, CA, 1992.

Sandberg, J. and Oerlemans, J.: Modelling of Pleistocene European ice sheets: The effect of upslope precipitation, Geologie en Mjinbouw, 62, 267-273, 1983.

Sellers, W.: A global climatic model based on the energy balance of the Earth-atmosphere system, J. Appl. Meteorol., 8, 392-400, 1969.

Svirezhev, Y. M. and von Bloh, W.: Climate, vegetation, and global carbon cycle: the simplest zero-dimensional model, Ecological Modeling, 101, 79-95, 1998.

Wang, W., Zhang, Q., Easterling, D., and Karl, T.: Beijing cloudiness since 1875, J. Clim., 6, 1921-1927, 1993.

Watson, A. and Lovelock, J.: Biological homeostasis of the global environment: the parable of Daisyworld, Tellus, 35B, 284-289, 1983.

Weber, S.: On homeostasis in Daisyworld, Climatic Change, 48, 465-485, 2001. 